\title{
DEPENDENCE OF THE NORTH-SOUTH COMPONENT OF IMF ON THE SOLAR WIND ACCELERATION
}

\author{
FELIX PEREIRA B. \\ Department of Physics, St. Xavier's College \\ Thumba, Trivandrum, India \\ and \\ GIRISH T. E. \\ Department of Physics, University College, \\ Trivandrum, India-695034.
}

September 6, 1996

\begin{abstract}
The monthly probability of occurrence of southward $\left(B_{z}^{-}\right)$component of IMF estimated independent of the sector polarity observed near earth is found to change with the magnitude of solar wind velocity. The above analysis is done for each month during two years around sunspot minima and maxima in cycle 21 . The results will be interpreted in terms of association of southward $B_{z}$ events with solar wind flows of distinct solar origin such as low and high speed solar wind.
\end{abstract}

\section{Introduction}

Solar origin of southward component of IMF $\left(B_{z}^{-}\right)$observed near earth is one of the crucial problems in solar terrestrial physics. Long term statistical studies of solar wind observations near $1 \mathrm{AU}$ have not reported any significant correlation between southward component of IMF and solar wind speed. Studies on the solar origin of southward component of IMF near earth for specific events such as major geomagnetic storms suggested (Lundstedt, 1991) three principal solar causes for the same.

1. Transient solar features such as CMEs

2. Coronal holes (Alfvenic fluctuations)

3. Heliospheric current sheet crossings (meridional deflections)

These phenomena are related to three distinct types of solar wind flows such as low speed, high speed and transient related. In this study, we report how the monthly probability of occurrence of southward component of IMF observed near earth varies with solar wind speed. We have considered two periods, one around sunspot minimum (1975-76) and another around sunspot maximum (1979-80) during solar cycle 21. We have used GSE system for our analysis since the occurrence of southward component is independent of the IMF sector polarity (east-west component or $B_{y}$ ) in this system. 
Fig. 1. Probability of observing southward component of IMF $\left(P_{s}\right)$ in different solar wind speed ranges for (a) 1975 September and (b) for 1979 June

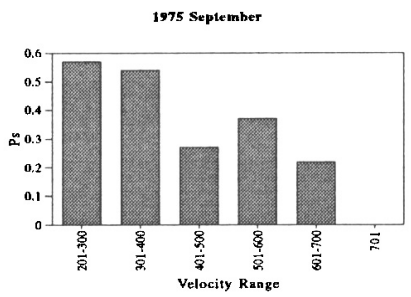

(a)

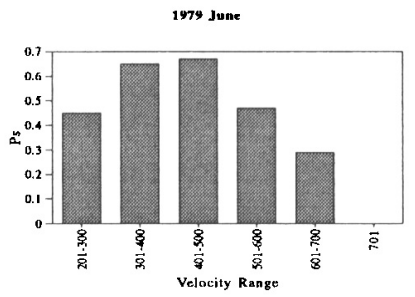

(b)

\section{Method of Data Analysis}

Published hourly values of observed solar wind velocity and magnetic field parameters in the GSE system by earth-orbiting satellites (King, 1979; Couzens and King, 1986) is used for our study. We have separated the hourly values of IMF data according to the following ranges of solar wind speed $201-300 \mathrm{~km} \mathrm{~s}^{-1}, 301-400 \mathrm{~km} \mathrm{~s}^{-1}, 401-500 \mathrm{~km} \mathrm{~s}^{-1}, 501-600 \mathrm{~km} \mathrm{~s}^{-1}$, $601-700 \mathrm{~km} \mathrm{~s}^{-1}$, and $\geq 701 \mathrm{~km} \mathrm{~s}^{-1}$. For these solar wind speed ranges we have estimated separately the probability of occurrence of southward component of IMF defined as $P_{s}$ for each month (January, February, etc.) during the years 1975, 76, 79 and 1980. (For these years solar wind plasma and magnetic field observations are available for most of the time.) A graphical representation of $P_{s}$ for each velocity range for the months September, 1975 and June 1979 is shown in Fig. 1.

We have also computed $P_{s}$ for the following two categories of solar wind speed i.e.,

(a) high speed $V>500 \mathrm{~km} \mathrm{~s}^{-1}$ and

(b) low speed $V<500 \mathrm{~km} \mathrm{~s}^{-1}$.

We define a parameter $\Delta P=P_{s}$ (low speed) $-P_{s}$ (high speed) for a given month. The monthly variation of $\Delta P$ for the above years are shown in Fig. 2 .

\section{Discussion}

The major results from this study are:

(a) It is found that the probability of observation of southward component of IMF near $1 \mathrm{AU}$ (independent of IMF sector polarity) varies with the range of solar wind speed. 
Fig. 2. Difference in the probability of observing IMF $B_{z}^{-}$component for low speed and high speed solar wind $(\Delta P)$ for the months in 1975-76 and 1979-80

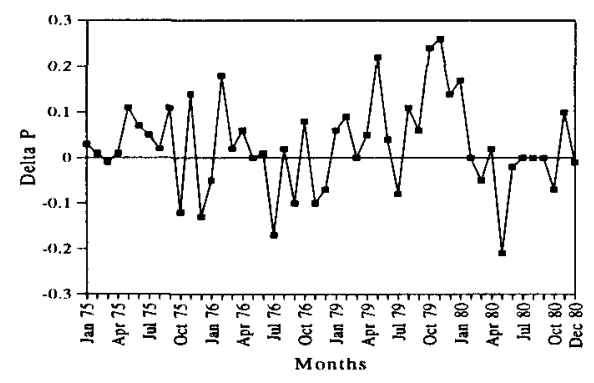

(b) We find a higher probability of occurrence of southward component of IMF associated with low speed solar wind compared to the same associated with high speed solar wind.)

(c) The above results do not show any significant difference between sunspot minima and sunspot maxima during cycle 21 .

The above results can be explained in terms of the existence of separate longitude zones on the sun for the occurrence of active centres (related to sunspots) and coronal holes. Low speed solar wind and transient solar features (flares, CMEs, etc.) have a common solar origin related to active centres while high speed solar wind originate mostly from coronal holes generally separated from the former. We also expect the above longitudinal organisation of solar phenomena persisting throughout the sunspot cycle. If it is assumed that the principal solar cause of southward component of IMF near $1 \mathrm{AU}$ as the solar transients, our results could be explained using the above model. The observation of higher values of $\left|B_{z}\right|$ associated with low speed solar wind compared to the same with high speed solar wind reported recently by Bruno et al., is in agreement with our findings. From a recent study related to heliomagnetic latitudinal distribution of CMEs, it is found that CMEs occur close to the HCS even though the distribution is different in sunspot minima and maxima (Mendoza and Enriquez Perez, 1993). 


\section{References}

Bruno, R., Villante, U., and Stecca, A. 1994. Ann. Geophysicae. 12, 105.

Couzens, D. A., and King, J. H. 1986. Interplanetary Medium Data Book Suppl. 3., NSSDC, NASA, Goddard Space Flight Center, Maryland.

King, J. H., 1979. Interplanetary Medium Data Book, NSSDC, NASA, Goddard Space Flight Center, Maryland.

Lundstedt, H., 1992. Planet Space Sci. 40, 457.

Mendoza, B., and Enriquez, R. P. 1993. J. Geophys. Res., 98, 9365. 